\title{
Tricholeiperia peruensis n. sp. (Nematoda, Molineidae) del quiróptero Lophostoma silvicolum occidentalis (Phyllostomidae) en Tumbes, Perú
}

\author{
Tricholeiperia peruensis $\mathrm{n}$. sp. (Nematoda, Molineidae) from Lophostoma \\ silvicolum occidentalis (Chiroptera, Phyllostomidae) in Tumbes, Peru
}

\author{
Marina Vargas ${ }^{1}$, Rosa Martínez ${ }^{2}$, Manuel Tantaleán ${ }^{3}$, Richard Cadenillas ${ }^{4}$ y Víctor \\ Pacheco $^{4}$
}

1 Facultad de Ciencias Biológicas, Universidad Nacional Mayor de Universidad Nacional Mayor de San Marcos, Cludad Universitaria, marina_vc243@hotmail.com.

2 Laboratorio de Parasitología de Fauna Silvestre, Facultad de Ciencias Biológicas, Universidad Ciencias Blologicas, Universidad Cón. (1) Per Lima 11, Perú. E-mail rmartinezr@ unmsm.edu.pe.

3 Laboratorio de Parasitología. Facultad de Medicina Veterinaria. Univerd Heredia E-mail mtantalean antaleanv@ hotmail.com

4 Departamento de Mastozoología. Museo de Historia Natural, Universidad Nacional Mayor de San Marcos.

Presentado: $15 / 11 / 2008$ Aceptado: 07/12/2008 Publicado online: 26/02/2009

\section{Resumen}

En el presente trabajo, se describe a Tricholeiperia peruensis $\mathrm{n}$. sp. (Nematoda, Molineidae) en base a 25 especímenes colectados del intestino de 5 murciélagos de la especie Lophostoma silvicolum occidentalis de la localidad de Angostura, distrito Pampas de Hospital, departamento de Tumbes, Perú. La nueva especie se caracteriza por la morfología de la vesícula cefálica en forma de capuchón con escotaduras, por el tamaño y forma de las espículas cuyo extremo distal de la rama externa se divide en dos, de las cuales la rama interna sufre una nueva división; por la disposición de las costillas de la bolsa copulatríz donde, entre otros detalles, las antero y postero-ventrales están separadas y las externo-dorsales nacen cerca de la parte media del tronco dorsal y son gruesas.

Palabras clave: Tricholeiperia peruensis, Nematoda, Molineidae, Lophostoma silvicolum occidentalis, Phyllostomidae, Tumbes, Perú.

\section{Abstract}

In the present work, Tricholeiperia peruensis. $\mathrm{n}$ sp. (Nematoda, Molineidae) is described on the basis of $25 \mathrm{col}-$ lected specimens from 5 bat intestines of the Lophostoma silvicolum occidentalis species from Angostura, district of Pampas de Hospital, department of Tumbes, Peru. The new species is characterized by the morphology of the cephalic vesicle in the form of hood with recesses, for the size and form of spikes whose distal end of the external branch is divided in 2 from which the internal branch undergoes a new division; by the disposition of the ribs of the copulatory apparatus where, among others details, the pre- and post ventral ribs are separated and the external-dorsal ribs begin near the half of the dorsal trunk and are heavy.

Keywords: Tricholeiperia peruensis, Nematoda, Molineidae, Lophostoma silvicolum occidentalis, Phyllostomidae, Tumbes, Peru.

\section{Introducción}

El Perú es un país que tiene una gran diversidad de quirópteros (Hice et al. 2004) ocupando el segundo lugar en el neotrópico con 8 familias, 61 géneros, y más de 160 especies (Pacheco et al. 2007; Pacheco 2008); sin embargo, es muy poco lo que se conoce de su fauna parasitaria. Freitas \& Ibañez (1963) describieron a Ochoterenatrema fraternum del estómago e intestino de Tadarida brasiliensis del departamento de La Libertad; posteriormente, Miranda (1965) estudió tremátodos y nemátodos también de Tadarida brasiliensis pero de la ciudad de Lima, anotando el hallazgo de Prosthodendrium scabrum (Caballero, 1940) Caballero,1943 y de Mollinostrongylus delicatus (Schwartz, 1928), desde entonces se han publicado, de manera esporádica, los resultados de algunas investigaciones realizadas en los departamentos de Ica (Mendoza et. al. 1997), Amazonas (Vaucher 1986), Loreto (Durette-Desset \& Vaucher 1988, 1989; Vaucher \& DuretteDesset 1999) y Cusco (Guerrero et al. 2002).

En el presente trabajo, se describe a Tricholeiperia peruensis n. sp. (Nematoda, Molineidae) colectada del intestino delgado de Lophostoma silvicolum occidentalis (Familia Phyllostomidae) procedente de la localidad de Angostura, distrito Pampas de Hospital provincia de Tumbes en el departamento de Tumbes.

\section{Materiales y métodos}

En setiembre del 2006, se capturaron cinco especímenes de Lophostoma silvicolum occidentalis (Fam. Phyllostomidae) utilizando redes de niebla en la localidad de Angostura correspondiente al bosque seco ecuatorial, ubicado a $03^{\circ} 45^{\prime} 23^{\prime \prime} \mathrm{S}$, $80^{\circ} 23^{\prime} 15^{\prime}$ 'W y $74 \mathrm{~m}$ de altitud en el distrito Pampas de Hospital de la provincia de Tumbes. Se colectaron 25 especímenes de nemátodos del intestino delgado, que fueron fijados en alcohol de $70 \%$. Para estudiarlos, se transparentaron en una mezcla de alcohol-fenol. Para examinar las espículas, previamente se disectaron seis machos y las espículas obtenidas se montaron en medio de Berlesse. El synlophe se estudió seccionando transversalmente un macho y una hembra a la altura de la parte media del cuerpo, según recomendación de Durette-Desset (1983, 1985). Las medidas se expresan en milímetros anotando entre paréntesis el rango. Los dibujos se hicieron con la ayuda de una cámara lúcida Carl Zeiss. Los especímenes se encuentran depositados en la Colección helmintológica del Museo de Historia Natural de la UNMSM (MUSM).

\section{Resultado}

\section{Tricholeiperia peruensis n.sp.}

(Figs 1-9)

Descripción: basada en cinco machos y cinco hembras.

\section{Macho}

Mide 6,525 (6,225-7,6) con un ancho máximo de 0,134 $(0,11-0,14)$ en la parte media del cuerpo, ancho mínimo de $0,052(0,05-0,06)$ cerca de la región cefálica. El capuchón cefálico, con una escotadura ventral y otra dorsal, mide 0,05 de largo por 0,076 (0,07-0,09). Esófago muscular con 0,44 de largo $(0,41-0,54)$. El anillo nervioso se ubica a 0,07 respecto a la región anterior (sólo se observó en un espécimen). No se 


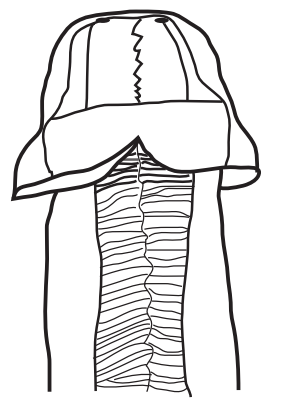

(1)

(2)

(3)

$0,04 \mathrm{~mm}$

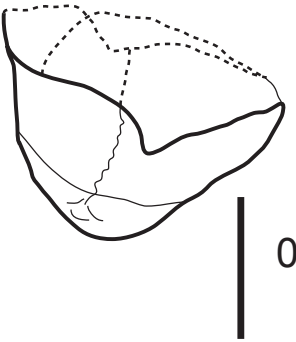

D

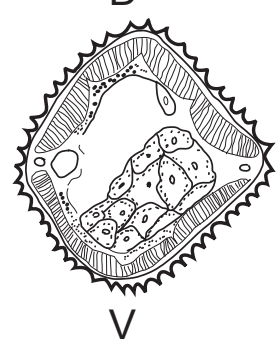

$\varepsilon$
$\varepsilon$
ठ
o

(5)

(6)

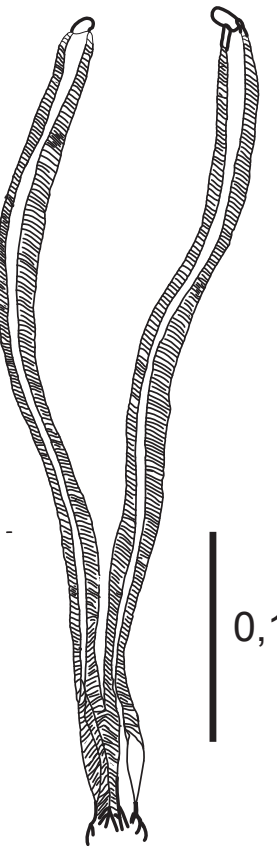

(4)
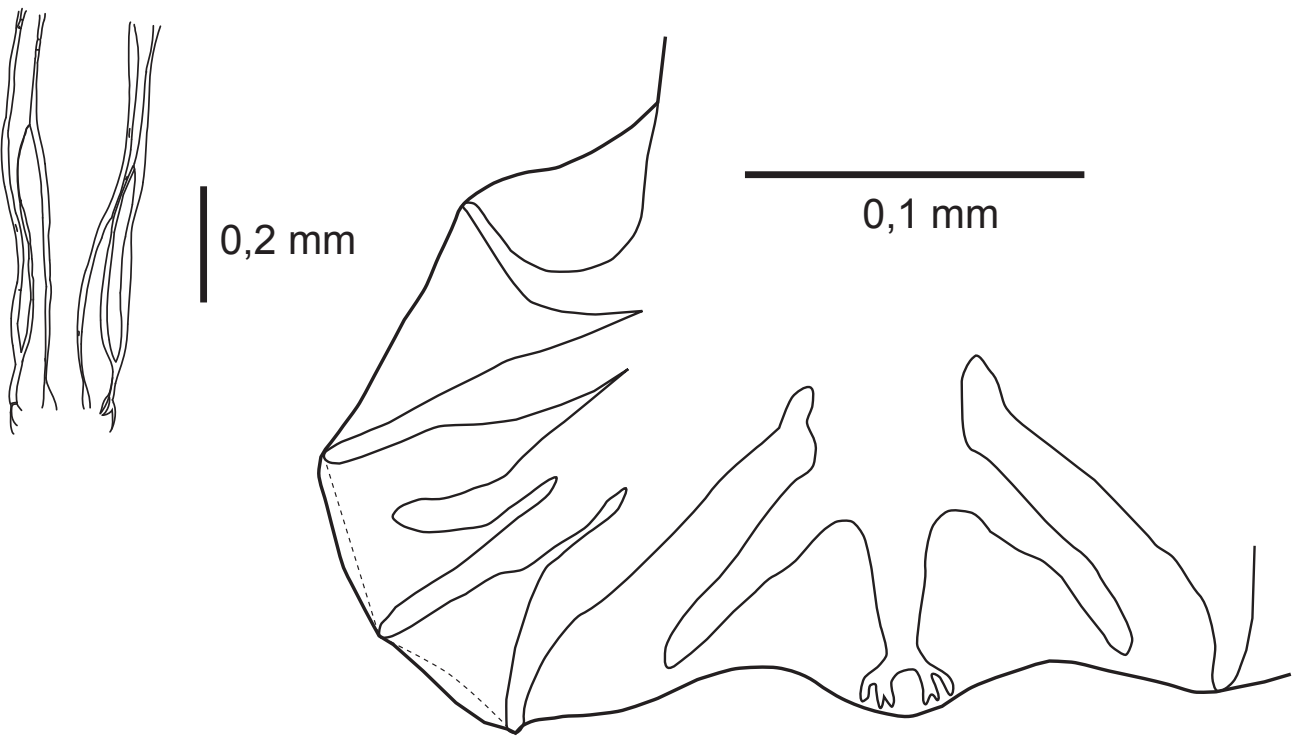

Figuras 1-6. Tricholeiperia peruensis n. sp. Macho adulto. 1. Región cefálica. 2. Corte del extremo cefálico mostrando las escotaduras dorsal y ventral. 3. Corte transversal en la parte media de la región corporal, mostrando el synlophe. 4. Espículas. 5. Extremo distal de las espículas. 6. Bolsa copulatriz mostrando la disposición de las costillas.

observó el poro excretor. El synlophe, uniforme en la parte media del cuerpo, está formado de 21 crestas dorsales y 25 ventrales.

La bolsa copulatríz tiene dos grandes lóbulos laterales y uno dorsal más pequeño; las costillas están dispuestas de la siguiente manera: las antero-ventrales y postero-ventrales están separadas y nacen de un tronco común, siendo las primeras curvadas anteriormente; las laterales también nacen de un tronco común, donde las antero-laterales son más cortas y están fusionadas con las medio-laterales hasta cierta longitud, luego se separan curvándose anteriormente pero sin llegar al borde de la bolsa; las postero-laterales inicialmente se encuentran ligeramente separadas de las medio-laterales pero después se separan realizando un leve quiebre. Las externo-dorsales son gruesas, ligeramente curvadas y paralelas con las postero-laterales. Cada rama de la costilla dorsal se divide en dos pequeñas ramas, de las cuales solo la interna vuelve a sufrir una nueva división dando dos ramas pequeñas.

Las espículas son de igual longitud, miden 0,472 (0,41$0,56)$ llevan alas que se fusionan en la parte distal y se bifurcan en ramas externa e interna, la primera se divide en el extremo distal en dos pequeñas ramas de las cuales una de ellas vuelve a subdividirse; la rama interna solo se divide una vez en el extremo distal.

\section{Hembra}

Mide 7,930 (6,087-9,025) de longitud con un ancho máximo de $0,195(0,11-0,24)$ a nivel de la región de la vulva. El capuchón cefálico tiene una longitud de 0,052 (0,05—0,058) por un ancho de 0,08 .

El poro anal se abre a $0,119(0,102-0,138)$ del extremo posterior. La región caudal presenta dos papilas y termina en una espina delgada de $0,039(0,0375-0,04)$ rodeada de otras tres espinas gruesas, dos ventrales y una dorsal, las cuales varían en longitud: la primera ventral de $0,034(0,025-0,037)$, la segunda de $0,034(0,025-0,041)$ y la dorsal de $0,088(0,022-0,0325)$. El synlophe es uniforme y compuesto de 18 crestas dorsales y 20 ventrales espaciadas regularmente a la altura de la parte media del cuerpo; se pudo contar de 11 a 12 crestas a nivel del ano.

Esófago muscular $0,432(0,41-0,46)$ de longitud. No se observó el anillo nervioso.

Vulva ubicada a 5,82 $(4,45-6,4)$ del extremo anterior y a $2,15(1,64-2,62)$ del posterior, con dos labios musculares de diferente longitud, el anterior de $0,068(0,065-0,07)$ y el posterior de $0,088(0,082-0,1)$. El vestíbulo presenta 2 ramas 


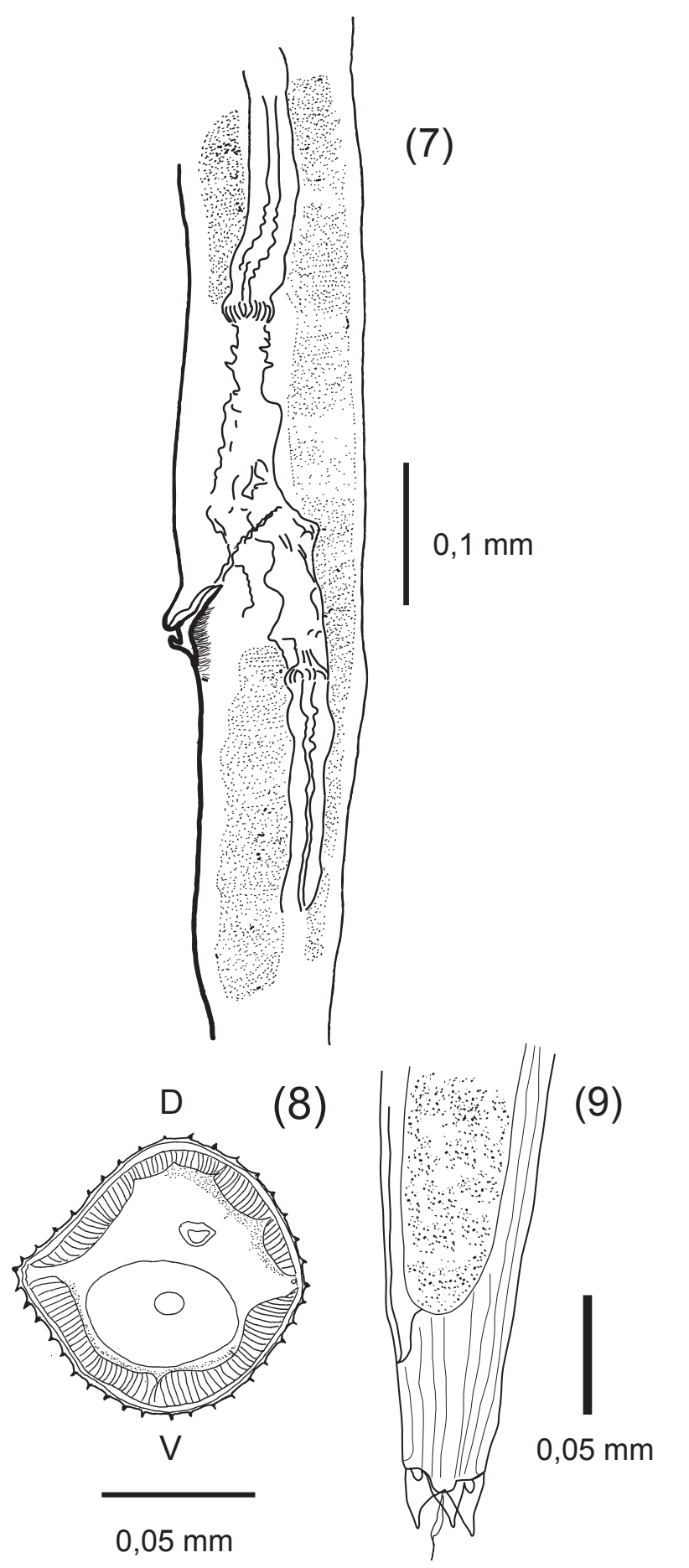

Figuras 7-9. Tricholeiperia peruensis n. sp. Hembra adulta. 7. Región vulvar en vista lateral, mostrando el vestíbulo con ramas desiguales. 8. Corte transversal en la parte media de la región corporal, mostrando el synlophe. 9. Región posterior en vista ventral.

de desigual longitud, la rama anterior, más grande, mide 0,216 $(0,188-0,25)$ y la rama posterior $0,139(0,142-0,162)$; los esfínteres son de igual longitud y miden 0,05 x 0,053 de ancho. Huevos ovalados no embrionados y de cáscara delgada, miden, dentro del útero, 0,097 de largo por 0,058 de ancho.

Huésped: Lophostoma silvicolum occidentalis (Fam. Phyllostomidae).

Localización: Intestino delgado.

Localidad: Angostura, Distrito Pampas de Hospital, provincia de Tumbes, departamento de Tumbes, Perú.
Holótipo: macho:MUSM N²773

Alótipo: hembra:MUSM N²774

Paratipo: MUSM N²775

\section{Discusión}

El género Tricholeiperia Travassos, 1935 se caracteriza por la forma de la vesícula cefálica, por la división en el extremo distal de las espículas, tamaño y disposición de las costillas de la bolsa copulatríz en el macho, longitud desigual de los vestíbulos y presencia de tubérculos en la cola de la hembra (Gibbson y Omah, 1991).

En América se han descrito 7 especies de Tricholeiperia: $T$. poeyi Barus \& Valle, 1967 de Tadarida (Mormopterus) minuta, Cuba; T. proencai Travassos, 1937 de Noctilio leporinus, Brasil; T. leiperi Chitwood, 1938 de Trachops cirrhosus, Brasil; T. carnigiensis Chitwood, 1938 de Natalus mexicanus, México; T. trinidadensis Gibbons \& Omah, 1991 de Natalus tumidirostris haymani, Trinidad; T. pearsei Chitwood, 1938 de Natalus mexicanus, México y Tricholeiperia sp. de Natalus (Nyctiellus) lepidus, Cuba; de ellas, solo Tricholeiperia trinidadensis es la que tiene mayor semejanza con Tricholeiperia peruensis $\mathrm{n}$. sp. en la ramificación de la costilla dorsal de la bolsa copulatríz, pero difieren en el mayor tamaño del cuerpo de nuestros especímenes (machos 6-7 mm vs. 2,6-3 $\mathrm{mm}$; hembras 6 $-7 \mathrm{~mm}$ vs. $4-5$ $\mathrm{mm}$ ) y consecuentemente de las diversas estructuras (vesícula cefálica, esófago, vulva, espículas y bolsa copulatriz); las espículas también son diferentes, pues en T. trinidadensis terminan en dos divisiones, en cambio en nuestro espécimen el extremo distal de la rama externa termina en dos divisiones de las cuales, la interna, sufre una nueva división originando 2 ramas de diferente longitud con una rama interna que continúa bifurcándose. Otras diferencias se aprecian en la forma y disposición de las costillas de la bolsa copulatríz; en efecto, mientras que en T. trinidadensis las costillas externo-dorsales nacen de la base anterior del tronco dorsal, son delgadas, curvadas y largas en $T$. peruensis nacen poco antes de la parte media del tronco dorsal, son gruesas y la curvatura no es pronunciada; además, en T. trinidadensis cada rama de la costilla dorsal se divide en dos, de las cuales una se vuelve a dividir en dos ramas de diferente longitud, en cambio, en la nueva especie esta subdivisión origina 2 ramas muy pequeñas de igual longitud. También, en T. trinidadensis las costillas antero y postero ventrales están fusionadas hasta una cierta longitud y la antero y medio laterales están fusionadas en gran parte de su trayecto, lo que no ocurre en nuestro espécimen pues, en ambos casos, se encuentran separadas. Otro carácter morfológico que diferencia ambas especies es el synlophe a nivel de la parte media del cuerpo del macho y de la hembra, porque T. trinidadensis tiene 25 crestas de diferente tamaño (14 dorsales y 11 ventrales) y 32 crestas (15 dorsales y 17 ventrales) respectivamente; y en nuestro espécimen, por el contrario, se observan 46 crestas en el macho ( 21 dorsales y 25 ventrales) y 38 crestas en la hembra (18 dorsales y 20 ventrales) en ambas de igual tamaño.

Tricholeiperia peruensis n. sp. también se diferencia de las otras cinco especies americanas. Así, difiere de Tricholeiperia poeyi y $T$. proencai por la forma de la vesícula cefálica y los detalles de la costilla dorsal, además carece del nódulo en la parte posterior de la bolsa copulatríz que presenta $T$. proencai; de $T$. carnigiensis y $T$. pearsei principalmente por las espículas que no son puntiagudas, además, por el mayor tamaño de la espículas 
de $T$. pearsei $(0,8 \mathrm{~mm})$. Finalmente, aunque no se tiene una descripción morfológica detallada de T. leiperi, esta difiere de la nuestra por el mayor tamańo del cuerpo $(9,3-11,5 \mathrm{~mm})$ por lo que es la especie de mayor tamaño hasta la actualidad.

Por todas estas consideraciones, creemos que nuestro espécimen corresponde a una nueva especie la que denominamos como Tricholeiperia peruensis por haberse encontrado en el Perú.

\section{Agradecimientos}

Los autores agradecemos a Liz Huamani, Lisseth Saenz, Mónica Alzamora, Carlos Mendoza, Jael Odar y Juan Carlos Jordán por participar en la colecta de los quirópteros. Esta investigación ha sido realizada gracias a los proyectos de investigación de los profesores Rosa Martínez y Víctor Pacheco del Instituto de Investigación en Ciencias Biológicas Antonio Raimondi (ICBAR) y Consejo Superior de Investigación, UNMSM (Cod. N71001231 y N061001021); y de Richard Cadenillas Ordinola (Proyectos de la Asociación Peruana para la Conservación de la Biodiversidad, APECO N 16-2006) y Bat Conservation International (BCI)).

\section{Literatura citada}

Barus V. \& M. T. del Valle. 1967. Systematic survey of nematodes parasitizing bats (Chiroptera) in Cuba. Folia Parasitológica, 14: 121-140.

Durette-Desset M. C. 1983. Keys to genera of the superfamily Trichostrongyloidea. In: Anderson, R.C. Chabaud. A.G. \& Willmott, S. (eds). CIH keys to the nematode parasites of vertebrates. Farnham Royal. UK. Commonwealth Agricultural Bureaux. $\mathrm{N}^{\circ} 10.86 \mathrm{pp}$.

Durette-Desset M.C. 1985. Trichostrongyloid nematodes and their vertebrate host: Reconstruction of the phylogeny of a parasitic group. Advances in Parasitology, 24: 239-306.

Durette-Desset M. C. \& C. Vaucher. 1988. Trichostrongyloidea (Nematoda) parasites de Chiroptères néotropicaux. II. Nouvelles données sur le genre Cheiropteronema Sandground, 1929. Revue Suisse Zool., 95: 889-899.

Durette-Desset M. C. \& C. Vaucher. 1989. Trichostrongyloidea (Nematoda) parasites de Chiroptères néotropicaux. III. Carostrongylus touzeti gen. n., sp. n. chez Carollia spp. (Phyllostomatidae). Revue Suisse Zool., 96: 697-706.
Freitas J.F.T. \& N. Ibañez. 1963. Fauna helmintológica do Perú, Ochoterenatrema fraternum sp. n. (Trematoda, Lecithodendriidae). Revista Brasileira de Biología, 23: 255-258.

Gibbons L. \& I. Omah. 1991. Tricholeiperia trinidadensis n. sp.(Nematoda, Molineidae) from Natalus tumidirostris haymani (Microquiroptera, Natalidae) in Trinidad. Systematic Parasitology, 20: 149-154.

Guerrero R., M. Coralie, L. Gardner, \& O. Bain. 2002. New and known species of Litomosoides (Nematoda: Filaroidea): Important adult and larval characters and taxonomic changes. Comparative Parasitology, 69: 177-195.

Hice C. L., P. M. Velazco, \& M. R. Willig. 2004. Bats of the Reserva Nacional Allpahuayo-Mishana, northeastern Peru, with notes on community structure. Acta Chiropterologica 6:319-334.

Mendoza L., J. Chávez \& M. Tantaleán. 1997). Cestodos parásitos de murciélagos de Ica, Perú. Parasitol al Día, 20: 20 - 24.

Miranda E. 1965. Contribución al conocimiento de la fauna helmintológica de Tadarida brasiliensis y descripción de Mollinostrongylus delicatus Schwartz, 1928. Tesis Bachiller Ciencias Biológicas. Univ. Nac. Mayor de San Marcos. Lima, 26 pp.

Pacheco V., R. Cadenillas, S. Velazco, E. Salas \& U. Fajardo. 2007. Noteworthy bat records from the Pacific Tropical rainforest region and adjacent dry forest in northwestern Peru. Acta Chiropterologica, 9: $409-422$.

Pacheco V. 2008. Lista de mamíferos del Perú, un avance que se presenta en el I Congreso de la sociedad peruana de Mastozoología,Cusco-Perú. http.//www.geocities.com/ vrpacheco/Lista Mamíferos del Peru. pdf.

Vaucher C. 1986. Cestodes parasites de Chirotères en Amerique du Sud. II. Hymenolepis mazanensis n. sp. Chez Saccopteryx bilineata (Temm.) et Rhynchonycteris naso (WiedNeuwied) (Chiroptera: Emballonuridae) en Amazonie péruvienne. Rev. Suisse Zool., 93: 817-821.

Vaucher,C. \& M . C. Durette-Desset. 1999. Histiostrongylus spinus n. sp. (Nematoda: Trichostrongylina), parásite de Phyllostomus discolor (Chiroptera: Phyllostomidae) et nouvelles dones sur les genres Histiostrongylus Molin, 1861 et Parahistiostrngylus Perez Vigueras, 1941. Revue Suisse Zool., 106: 611-620. 